\title{
Multifocal Cholesterol Granulomas of the Thymus: A Case Report of Positron Emission Tomography- Positive Benign Tumor of the Anterior Mediastinum
}

\author{
Mikito Suzuki, MD, ${ }^{1}$ Hirotoshi Horio, MD, PhD,${ }^{1}$ Azusa Yamada, MD, ${ }^{1}$ Reiko Shimizu, MD, ${ }^{1}$ \\ Toshiyuki Shima, MD, PhD, ${ }^{1}$ Masahiko Harada, MD, PhD, ${ }^{1}$ and Tsunekazu Hishima, $\mathrm{MD}^{2}$
}

\begin{abstract}
Cholesterol granulomas of the thymus are extremely rare benign entities. Herein, we describe a case of cholesterol granuloma in a 45 -year-old man who presented with multifocal anterior mediastinal lesions on computed tomography (CT). Positron emission tomography (PET)-CT revealed increased fluorodeoxyglucose uptake, with a maximum standardized uptake value of 8.3. Diffusion-weighted magnetic resonance imaging (MRI) revealed extreme hypointensity. He underwent total thymectomy by median sternotomy for presumed multiple thymoma. On histopathological analysis, cholesterol granuloma of the thymus was confirmed, and the patient had an uneventful postoperative course without recurrence for $\mathbf{2 8}$ months. Cholesterol granuloma of the thymus is a PET-CT-positive benign tumor with unique histological findings. The MRI findings were indicative of hypocellularity associated with a benign entity, whereas the PET-CT findings corresponded to granulomatous inflammation. Therefore, concurrent use of PET-CT and MRI can be helpful in distinguishing between benign cholesterol granulomas and malignant anterior mediastinal tumors.
\end{abstract}

Keywords: cholesterol granuloma, thymus, anterior mediastinum, positron emission tomography, magnetic resonance imaging

\section{Introduction}

Cholesterol granulomas are benign conditions characterized by cholesterol clefts and multinucleated giant cells that commonly develop in the middle ear and

${ }^{1}$ Department of Thoracic Surgery, Tokyo Metropolitan Cancer and Infectious Diseases Center Komagome Hospital, Tokyo, Japan

${ }^{2}$ Department of Pathology, Tokyo Metropolitan Cancer and Infectious Diseases Center Komagome Hospital, Tokyo, Japan

Received: August 29, 2021; Accepted: October 17, 2021

Corresponding author: Mikito Suzuki, MD. Department of Thoracic Surgery, Tokyo Metropolitan Cancer and Infectious Diseases Center Komagome Hospital, 3-18-22 Honkomagome, Bunkyo-ku, Tokyo 113-8677, Japan

Email: msuzuki@cick.jp

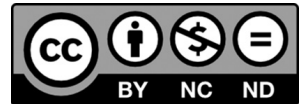

This work is licensed under a Creative Commons Attribution-NonCommercialNoDerivatives International License.

(C)2021 The Editorial Committee of Annals of Thoracic and Cardiovascular Surgery paranasal sinuses. ${ }^{1)}$ Cholesterol granuloma of the thymus is extremely rare, sometimes presenting as multifocal lesions in the anterior mediastinum. ${ }^{2,3)}$ Herein, we report a rare case of multifocal cholesterol granulomas of the thymus mimicking a thymic epithelial tumor.

\section{Case Presentation}

A 45-year-old man with an incidental finding of anterior mediastinal masses by routine computed tomography (CT) was referred for further investigation. His medical history included dyslipidemia and fatty liver disease. He had no history of trauma. Enhanced CT showed a 4.2-cm mass in the anterior mediastinum with slight contrast enhancement and spotty calcification, surrounded by 3 lesions measuring up to $0.8 \mathrm{~cm}$ (Fig. 1A). Contrast-enhanced magnetic resonance imaging (MRI) showed homogeneous hypointensity on both T1- and T2-weighted images without contrast enhancement (Fig. 1B). Diffusion-weighted 
Suzuki M, et al.
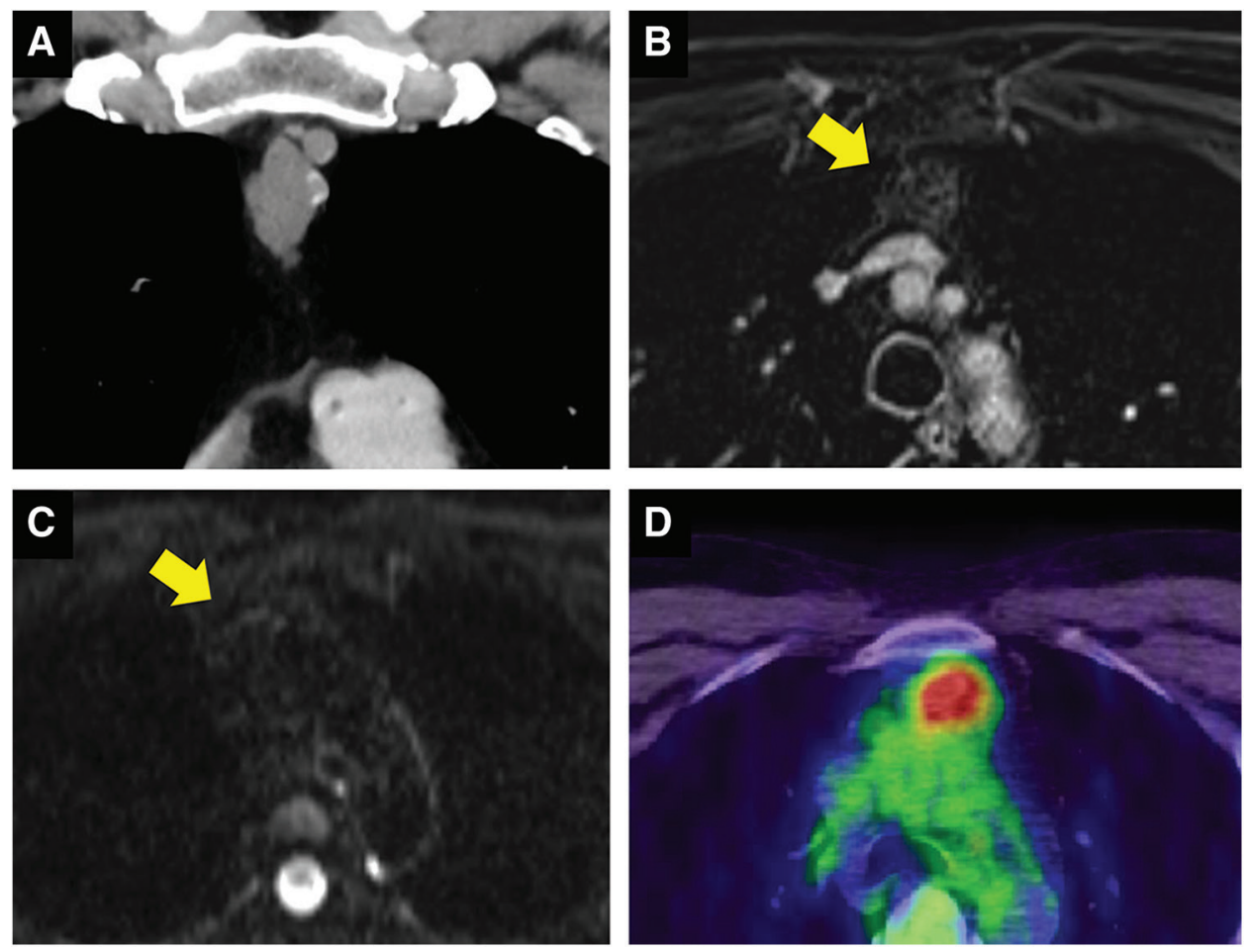

Fig. 1 Preoperative imaging findings. (A) Coronal enhanced CT scan. Multiple lesions up to $4.2 \mathrm{~cm}$ in the anterior mediastinum had slight contrast enhancement and spotty calcification. (B) Contrast-enhanced MRI. A homogeneous mass in the anterior mediastinum without contrast enhancement was presented (arrow). (C) DWI. The signal intensity was extremely low (arrow). (D) PET-CT. A high FDG uptake with a maximum standardized uptake value of 8.3 was revealed. CT: computed tomography; MRI: magnetic resonance imaging; DWI: diffusionweighted imaging; PET: positron emission tomography; FDG: fluorodeoxyglucose

imaging (DWI) revealed an image with extremely low signal intensity (Fig. 1C). Positron emission tomography (PET)-CT showed higher fluorodeoxyglucose (FDG) uptake in the main tumor with a maximum standardized uptake value of 8.3 (Fig. 1D). Total cholesterol $(240 \mathrm{mg} / \mathrm{dL})$ and triglyceride $(340 \mathrm{mg} / \mathrm{dL})$ levels were slightly elevated. Tumor markers, including carcinoembryonic antigen, alpha-fetoprotein, human chorionic gonadotropin, soluble IL-2 receptor, and anti-acetylcholine receptor antibody levels, were within normal ranges.

A highly aggressive thymic epithelial tumor was suspected from enhanced CT and PET-CT findings, and the patient consented to total thymectomy through a median sternotomy approach. Intraoperatively, there were firm and well-circumscribed masses in the left lobe of the thymus. The main tumor was $4.3 \times 3.0 \times 1.5 \mathrm{~cm}$ in size with a dark red cut surface, and small-sized nodules up to $1.0 \mathrm{~cm}$ were also seen. Microscopically, these tumors contained numerous cholesterol clefts surrounded by multinucleated giant cells and histocytes, which formed granulomas (Fig. 2A). Focal hemorrhage, hemosiderin deposition, calcification, and osseous metaplasia were also observed (Fig. 2B). There was no evidence of malignancy. Finally, a pathological diagnosis of cholesterol granuloma of the thymus was made. The patient's postoperative course was uneventful without recurrence for 28 months.

\section{Discussion}

Cholesterol granuloma is a benign mass containing cholesterol clefts and multinucleated giant cells that commonly develop in the middle ear and paranasal sinuses. ${ }^{1)}$ Cholesterol granuloma of the thymus is extremely rare, whose exact pathogenesis remains unclear but is thought to involve focal microhemorrhages via angiopathy resulting from trauma, chronic inflammation, or dyslipidemia, as in our patient. The angiopathy induces deposition of cholesterol clefts that stimulate a foreign-body giant-cell reaction and granuloma formation. ${ }^{1-6)}$

Cholesterol granulomas resemble thymic epithelial tumors on diagnostic imaging and can be difficult to detect preoperatively. We suspected a high-grade 

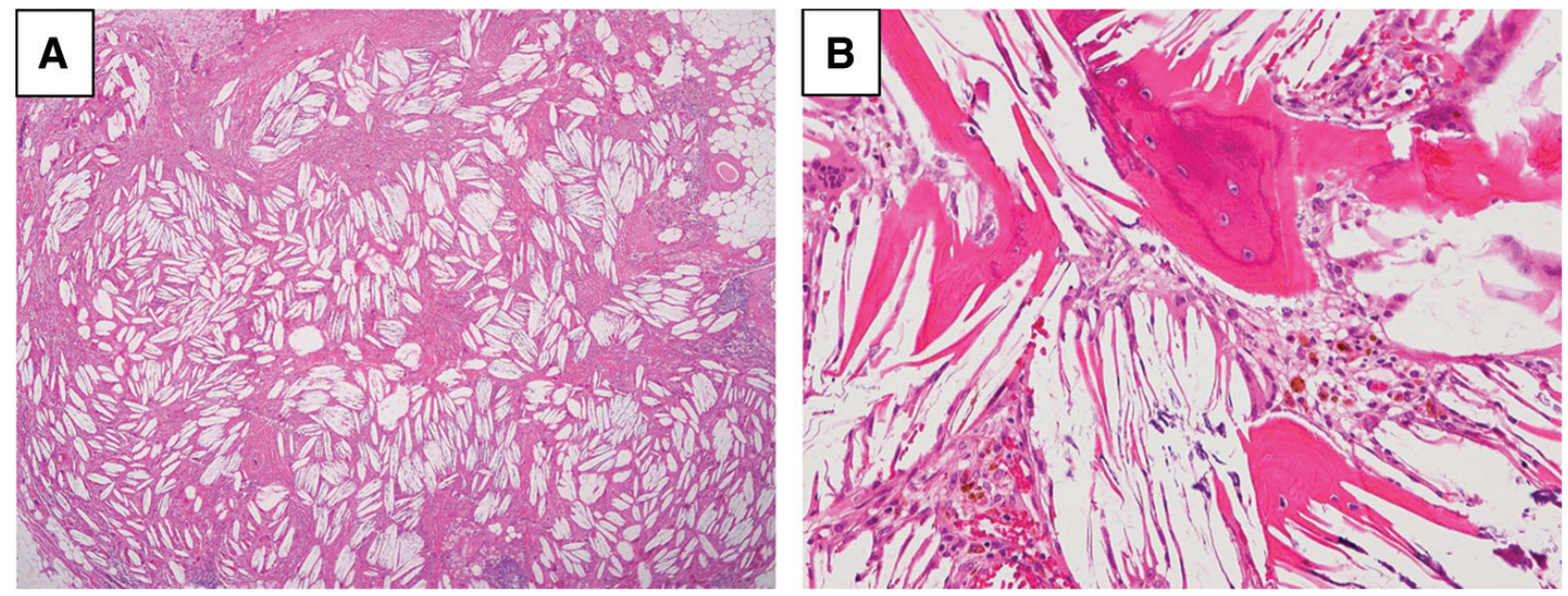

Fig. 2 Pathological findings. (A) The low-power view. Numerous cholesterol clefts with surrounding multinucleated giant cells and histocytes, which comprised a granuloma (hematoxylin-eosin staining). (B) The high-power view. Cholesterol clefts with hemorrhage, hemosiderin deposition, calcification, and osseous metaplasia (hematoxylin-eosin staining).

multiple thymoma considering the multifocal mediastinal lesions, and high FDG uptake on CT and PET-CT images, which is usually observed in hypercellular tumors and malignancies. However, it is interesting to note that benign inflammatory masses such as cholesterol granuloma of the thymus also show remarkable FDG uptake because of granulomatous inflammation. ${ }^{3-5)}$

MRI is used to distinguish benign from malignant mediastinal tumors. ${ }^{7)}$ The MRI features of cholesterol granulomas of the thymus have rarely been reported. ${ }^{5)}$ In the present case, extremely low signal intensity on both T1- and T2-weighted images reflected fiber, calcium, or hemosiderin. Cystic thymomas with chronic intratumoral hemorrhage can exhibit similar signals reflecting fibrosis, calcification, and hemosiderin. ${ }^{8)}$ Nevertheless, unlike our case, images with heterogeneous intensity are often seen in cystic thymomas. Furthermore, the lack of contrast enhancement and hypointensity on DWI indicated hypocellularity. Histologically, the tumor was composed of noncellular components, such as cholesterol clefts (Fig. 2A and 2B). This case demonstrates the value of MRI with the addition of PET-CT to differentiate cholesterol granulomas from malignant mediastinal tumors.

Multiple thymoma typically presents as multifocal anterior mediastinal tumors ${ }^{9,10)}$; however, two cases of cholesterol granuloma of the thymus presenting as multifocal lesions have been reported., ${ }^{2,3)}$ Therefore, cholesterol granulomas should be considered in the differential diagnosis of multiple anterior mediastinal tumors.

There have been no reports on cholesterol granulomas of the thymus diagnosed preoperatively. A preoperative biopsy is difficult for anatomical reasons, ${ }^{2,3)}$ and the presence of cholesterol clefts on histology is insufficient to confirm the diagnosis because cholesterol clefts can also be found in thymic cysts or thymomas. ${ }^{6}$ As cholesterol granulomas do not recur after complete resection, ${ }^{2,3,5,6)}$ surgical resection and pathological evaluation are important for the proper diagnosis and treatment of this unique tumor.

\section{Conclusion}

Herein, we report a case of multifocal cholesterol granulomas of the thymus. Cholesterol granuloma is a PET-positive benign tumor of the thymus that mimics a thymic epithelial tumor. Concurrent use of PET-CT and MRI can be helpful in distinguishing benign cholesterol granulomas from malignant anterior mediastinal tumors.

\section{Disclosure Statement}

The authors have no conflicts of interest.

\section{References}

1) Krishnan TR, Sinha SK, Kejriwal NK. A rare case of cholesterol granuloma in the anterior mediastinum. Heart Lung Circ 2013; 22: 303-4.

2) Manabe T, Oka S, Ono K. Multifocal cholesterol granulomas of the anterior mediastinum. Surg Case Rep 2020; 6: 182 .

3) Nagata S, Ishihara M, Omasa M, et al. Multifocal thymic cysts with cholesterol granuloma. Respirol Case Rep 2018; 6: e00361. 
Suzuki M, et al.

4) Drury NE, Smith DN, Phillips LM, et al. Cholesterol granuloma of the anterior mediastinum. Thorax 2017; 72: 671-2.

5) Fujimoto K, Takamori S, Yano H, et al. Focal cholesterol granuloma in the anterior mediastinum: [18F]-fluoro-2-deoxy-D-glucose-positron emission tomography and magnetic resonance imaging findings. J Thorac Oncol 2007; 2: 1054-6.

6) Weissferdt A, Kalhor N, Moran C. Primary thymic cholesteroloma: a clinicopathological correlation of four cases of an unusual benign lesion. Virchows Arch 2015; 467: 609-11.

7) Usuda K, Maeda S, Motono N, et al. Diffusion weighted imaging can distinguish benign from malignant mediastinal tumors and mass lesions: comparison with positron emission tomography. Asian Pac J Cancer Prev 2015; 16: 646975 .

8) Benveniste MF, Rosado-de-Christenson ML, Sabloff $\mathrm{BS}$, et al. Role of imaging in the diagnosis, staging, and treatment of thymoma. Radiographics 2011; 31: 1847-61; discussion 1861-3.

9) Mori T, Nomori H, Ikeda K, et al. Three cases of multiple thymoma with a review of the literature. Jpn J Clin Oncol 2007; 37: 146-9.

10) Kawaguchi K, Usami N, Uchiyama M, et al. Triple thymoma with different histologic types. J Thorac Cardiovasc Surg 2007; 133: 826-7. 